Palabra Clave (La Plata), abril 2017, vol. 6, n² 2, e021. ISSN 1853-9912

Universidad Nacional de La Plata.

Facultad de Humanidades y Ciencias de la Educación.

Departamento de Bibliotecología

\title{
Estudos métricos da informação em periódicos do Portal SciELO: visibilidade e impacto na Scopus e Web of Science
}

\author{
Metric studies of information in journals indexed at \\ SciELO: visibility and impact at Scopus and Web of \\ Science
}

\section{Juliana Lazzarotto Freitas *, Fabio Sampaio Rosas *, Sandra Edith Miguel $* *$}

* Universidade Estadual Paulista Júlio de Mesquita Filho. Faculdade de Filosofia e Ciências. Programa de Pós-Graduação em Ciência da Informação - (UNESP), Brasil; ** Universidad Nacional de La Plata. Facultad de Humanidades y Ciencias de la Educación. Instituto de Investigaciones en Humanidades y Ciencias Sociales - IdIHCS (UNLP-CONICET), Argentina I jlazzarotto@marilia.unesp.br, fabio@dracena.unesp.br, smiguel@fahce.unlp.edu.ar

\section{PALAVRAS CHAVE}

Indicador de citação

Impacto

Visibilidade

Produção periódica científica

Estudos métricos da informação

\section{KEYWORDS}

Citation indicator

Impact

Visibility

Scientific periodical production

Metric Studies of Information

\section{RESUMO}

Objetiva analisar a visibilidade dos periódicos que publicam pesquisas sobre estudos métricos da informação, a partir de indicadores de impacto presentes nas bases indexadoras Scopus e Web Of Science (WOS). Os periódicos selecionados representam o domínio de estudos métricos da informação na base SciELO e estão presentes nas outras referidas bases, das quais foram extraídos seus respectivos indicadores de citação. Identifica o número total de publicações de cada periódico e o número total de suas publicações relacionadas aos estudos métricos (EMI) no período de 2009 a 2013, e o número total de citações recebidas por cada periódico e suas citações aos artigos de EMI na janela temporal 2009-2016, evidenciando a média de citação por periódicos, áreas e países, na temática e geral. Logo após, compara as medidas de citação desses artigos, dadas pelo Scimago Journal \& Country Rank (SJR) da Scopus e pelo Journal Citation Reports (JCR) da WOS, apontando as revistas, áreas e países com maior impacto na temática e no geral. Conclui-se que, a maioria das revistas obtiveram maior quantidade de citações na Scopus em detrimento à WOS, sugerindo maior visibilidade na primeira para os artigos que tratam de estudos métricos. Situação semelhante ocorre quando se considera toda a produção científica das revistas e suas citações recebidas, em relação ao impacto geral que apresentam. Em termos relativos, observa-se que, em ambas as bases, as revistas de Ciências Sociais Aplicadas apresentam o maior percentual de artigos sobre EMI em detrimento às revistas de outras disciplinas. Por fim, considera que as políticas de indexação na base Scopus são responsáveis por sua cobertura mais ampla e pela maior visibilidade de seus periódicos, que estão disponíveis em diferentes idiomas.

\section{ABSTRACT}

This study aims to analyze the visibility of the journals that publish research on metric studies (MS) by using the impact indicators of these journals, present at the databases Scopus and Web of Science (WOS). The selected journals represent the domain metric studies in the SciELO database and are present in the other databases, which were taken from their citation indicators. The study identifies the total number of each journal and the total number of publications related to metric studies in the 2009-2013 period, and the total number of citations received by each journal and his quote to MS articles in the time window 2009-2016, showing the average quotation by journal, areas and countries in the thematic and in their general publication. Soon after, it compares the citation measures given by the SJR of Scopus and the JCR WOS, pointing journals, areas and countries with the greatest impact on the theme and overall. It concludes that the majority of the journals have a higher number of citations in Scopus over the WOS, suggesting greater visibility in the first database to the articles about with metric studies. A similar situation occurs when considering any scientific journal and their citations received in relation to their overall impact. In relative terms, it observes that, in both databases, the Applied Social Sciences journals presents the highest percentage of articles on EMI in detriment to the other disciplines journals. Finally, it considers that Scopus indexing policies are responsible for their wider coverage and greater visibility of their journals, which are available in different languages.

Recibido: 8 de febrero de 2017 | Aceptado: 18 de abril de 2017 | Publicado: 20 de abril de 2017 


\section{Introdução}

O campo de Estudos Métricos da Informação (EMI) caracteriza-se por seu caráter multidisciplinar, tanto em relação à procedência dos autores como dos periódicos nos quais esses autores publicam os resultados de suas pesquisas. Isto foi comprovado em diversos estudos, desde uma perspectiva geográfica global (Glänzel y Schoepflin, 1994; Schoepflin y Glänzel, 2001; Patra, Bhattacharya y Verma, 2006) como dimensionados a países específicos (Meneghini y Packer, 2010, Cabrini Grácio y Tannuri de Oliveira, 2012; Miguel y Dimitri, 2013). Apesar de uma parte importante da produção científica gerada no campo concentrar-se em revistas especializadas como a "Scientometrics", existem numerosos estudos dispersos em diferentes publicações de distintos domínios disciplinares. Essa referida multidisciplinaridade característica do campo é entendida como possibilidade de estabelecimento de relações interdomínios, e de realização de estudos que, desde uma perspectiva holística, contribuam a uma maior compreensão de seu desenvolvimento e evolução.

Buscando aprofundar pesquisa anterior sobre a produção periódica científica relacionada aos estudos métricos da informação, presente na base Scientific Eletronic Library Online (SciELO), cuja análise propiciou uma visão ampla da transversalidade desse domínio, distribuído em periódicos de distintos campos (Rosas y Freitas, 2016) pretende-se nesse estudo, analisar a visibilidade dos periódicos que publicam pesquisas sobre o tema, a partir de indicadores de impacto desses periódicos, presentes nas bases indexadoras Scopus e Web of Science (WOS).

Para tanto, objetiva-se evidenciar como se configura o espaço destinado aos estudos métricos da informação em diferentes campos do conhecimento, a partir da análise da representatividade do impacto dos artigos relacionados aos EMI para esses periódicos, e o impacto da totalidade de suas publicações, por meio da média de citação que apresentam. Para o alcance desse objetivo, identifica-se o número total de publicações de cada periódico e o número total de suas publicações relacionadas aos EMI no período de 2009 a 2013, e o número total de citações recebidas por cada periódico e suas citações aos artigos de EMI na janela temporal 2009-2016. Logo após, comparam-se as medidas de citação desses artigos, dadas pelo Scimago Journal \& Country Rank (SJR) da Scopus e pelo Journal Citation Reports (JCR) da WOS para as revistas que estão indexadas em ambas as bases.

\section{Referencial teórico}

O panorama internacional de periódicos científicos apresenta-se em contexto dinâmico, com características delineadas a partir das transformações nos modos de produção. A literatura sobre esse fenômeno é extensa, especialmente aquela voltada aos métodos de análise e avaliação dos periódicos. Ao apontar as dificuldades da aplicação de indicadores elaborados para um contexto a outro contexto, Porcaro (2005) visualiza as variáveis que esse problema apresenta com relação ao uso dos indicadores da Organisation for Economic Co-operation and Development (OCDE). Construídos para a sociedade industrial, eles sofrem problemas de adaptação ao atual contexto da Sociedade da Informação.

Similares dificuldades enfrentam os indicadores bibliométricos para avaliar o impacto da pesquisa. Numerosas reflexões e debates estão ocorrendo na própria comunidade científica sobre o tema, o que pode ser constatado em recentes documentos como a Declaração de São Francisco (DORA) em 2012 e o Manifesto de Leiden (Hicks et al. 2015). 
Apesar das questões elencadas, a citação é um dos indicadores amplamente aceitos na comunidade científica e no mundo acadêmico, especialmente por representar uma forma de explicitar o reconhecimento e a utilidade dos conhecimentos precedentes, sendo suas motivações e as diferentes perspectivas teóricas que a sustentam, amplamente documentadas (Nicolaisen, 2007; Bornmann y Hans-Dieter, 2008).

Sobre essa premissa, a quantidade de citações recebidas pelas publicações científicas é uma das medidas bibliométricas mais utilizadas para estimar o impacto dos resultados da investigação.

Neste trabalho, entende-se o impacto dos artigos como o reconhecimento pela comunidade científica por meio das suas citações recebidas e o impacto das revistas, como o reconhecimento alcançado pelas citações dos artigos publicados nelas. Já, a visibilidade entende-se como a difusão das revistas e dos artigos publicados nelas, ao serem indexadas nas bases de dados. Em suma, a visibilidade é a vitrine, que favorece que um artigo seja visto, lido e citado.

Logo, a citação é um indicador importante para aferir o impacto de determinado campo. Como já dito, embora esse indicador tenha sido legitimado para determinar a visibilidade de campos científicos distintos, é uma medida relativa ao contexto, que apresenta divergências relacionadas à busca por uma avaliação menos desigual da ciência, especialmente se relevadas as especificidades de cada campo. Segundo Mugnaini (2006), esse indicador não necessariamente remete à aferição da qualidade de uma pesquisa, representando o impacto restrito à uma realidade específica.

Ainda assim, no contexto das bases de dados indexadoras e da produção ali indexada, pode ser considerado como medida para analisar a visibilidade das pesquisas na comunidade científica.

Tannuri de Oliveira (2011), em uma análise do domínio de estudos métricos, trabalha com medidas de produtividade e de visibilidade para evidenciar o impacto e visibilidade das pesquisas relacionadas aos estudos métricos junto à comunidade científica. Segundo a autora, é importante entender e conhecer "a diversidade das áreas de Bibliometria e Cientometria", pois essa compreensão representa o passo fundamental "para unificar esforços na busca pela consolidação da identidade acadêmico científica desse campo.

\section{Metodologia}

O objeto aqui analisado foi inicialmente delimitado a partir de um recorte de periódicos relacionados aos estudos métricos da informação, resultante de estudo publicado anteriormente pelos autores, que partiu de uma busca temática na base SciELO com alguns termos relacionados ao domínio, em todos os índices, a saber: bibliometr*, scientometr*, informetr*, infometr*, webometr*, patentometr*, "scientific collaboration", co-authorship, "citation analysis", "co-citat*", "impact factor", "h index", "Bradford's law", "Zipf's law", "Lotkas' law", obsolescence, "scientific policy" e "metric studies". Não foi incluído o termo altmetr* pelo fato de ser um estudo continuação de outro anterior que não incluiu este novo termo e que seguiu uma estratégia de busca realizada a partir de termos sugeridos em estudos de Grácio e Oliveira (2012), que também se baseou em pesquisas anteriores de Meneghini e Packer (2010), Lu e Wolfram (2010) e Machado (2007) e que, em 2013, ainda não havia sido considerado como termo representativo e consolidado dentro do domínio. Entretanto, o novo termo será levado em conta para a realização de futuros estudos no 
domínio.

A intenção de selecionar periódicos na base SciELO visou valorizar a produção periódica científica da Iberoamérica e África do Sul, permitindo observar o impacto dessas revistas e da sua produção em estudos métricos em nível global a partir da Scopus e da WOS, já que não foi possível obter o indicador de impacto dos artigos do corpus na própria base SciELO, pois apesar da SciELO disponibilizar acesso a dados estatísticos de periódicos indexados em sua base de dados por meio do site SciELO Analytics, ainda em formato beta, não é possível verificar as citações recebidas por artigo, indicador utilizado por esse estudo para mensuração do impacto da produção científica em EMI.

Analisaram-se então, dez das 15 revistas identificadas na SciELO com a maior quantidade de artigos relacionados aos EMI, já que, somente estas estavam presentes na Scopus e na WOS. Nesse caso, a seleção inicial das revistas foi realizada na SciELO, em que totalizaram 15 delas. Entretanto, apenas dez das 15 foram usadas como corpus, porque estavam também indexadas na Scopus e na WOS. Portanto, a fim de que os resultados sobre a visibilidade fossem precisos e comparáveis, foram eliminadas deste estudo as seguintes revistas: Acimed (Cuba), Ciência da Informação (Brasil), Index de Enfermería (Espanha), Revista de Administração Contemporânea (Brasil) e Revista Interamericana de Bibliotecología (Colômbia).

As revistas analisadas foram: Gaceta Sanitaria (Gac. Sanit.); Interciencia-INCI (Interciencia); Investigación Bibliotecológica (Investig. Bibl.); Nutrición Hospitalaria (Nutr. Hosp.); Perspectivas em Ciência da Informação (Perspect. Ciênc. Inf.); Revista de Administração de Empresas (RAE); Revista de Saúde Pública (Rev. Saúde Pública); Revista Médica de Chile (Rev. Med. Chile); South African Journal of Science (S. Afr. J. Sci.); e Transinformação. Ressalta-se que a produção do ano de 2013 da revista Perspectivas em Ciência da Informação, indexada na base Scopus, foi retirada do corpus dessa pesquisa, por não estar indexada na base WOS, limitação metodológica que deve ser considerada para a análise dos resultados, quando do menor impacto apresentado por esse periódico.

Para identificar o impacto da produção periódica científica na temática, coletou-se a quantidade de citações recebidas pelos artigos indexados nas bases Scopus e WOS, que totalizaram 89 artigos.

Para efeito de comparação com essa produção coletada, referente à temática de estudos métricos, também foram coletados dados quantitativos da produção e citação total das revistas mencionadas. A última coleta foi realizada nas bases de dados Scimago Journal Rank (Scopus) e Journal Citation Report (WOS). Para a quantificação da produção foi estabelecido o período de 2009 a 2013, e para a quantificação das citações a janela temporal abarcou mais três anos, que considera-se ser tempo suficiente para o alcance da maturidade científica dos artigos publicados nos últimos anos do período analisado, estabelecendo, portanto a janela de citação de 2009 a 2016, a fim de que estudo das citações fosse mais representativo para este corpus. Não foi possível recortar os anos anteriores a 2009 porque as revistas estudadas não apresentavam sua produção completa indexada na base para os anos anteriores, interferindo, desse modo, na integridade da análise para cada base e para cada revista. Os dados de citações, tanto os relativos à produção na temática quanto à produção geral das revistas, foram coletados entre os dias 01 e 04 de julho de 2016.

A seguir, foram tabulados e organizados no software Excel para geração de gráficos e para o cálculo das médias de citação das revistas nas duas bases de dados, tanto para a produção total como para a temática, a fim de compará-las. 


\section{Resultados}

A coleta de dados foi organizada de modo a visualizar as revistas e suas médias de citação em cada base; as áreas em que estão inseridas segundo a divisão de áreas da SciELO e por fim, os países dos quais procedem.

A Tabela 1, ordenada pelo título das revistas, apresenta a produção científica geral das revistas analisadas durante o período de 2009 a 2013, bem como o total de citações por elas recebidas entre 2009 e 2016. Observa-se que a revista que mais produziu nas duas bases de dados analisadas, durante o período, foi a Revista Médica do Chile, da área da Saúde. Em contrapartida, a que mais foi citada nas duas bases foi a revista espanhola Nutrición Hospitalaria, também da Saúde. Entretanto, observa-se que, tanto na Scopus como na WOS as revistas de Ciências Sociais Aplicadas apresentam, em termos relativos, o maior percentual de artigos sobre EMI em detrimento às revistas de outras disciplinas.

A Investigación Bibliotecológica ocupa o primeiro lugar, seguida da Perspectivas em Ciência da Informação, Transinformação e logo a RAE, com menor presença. Claramente se evidencia que apesar do caráter multidisciplinar dos EMI, os três periódicos com a maior presença relativa de estudos sobre a temática são da Ciência da Informação.

Além disso, a Rev. Médica do Chile tem maior impacto na temática (Tabela 2) do que em suas publicações totais, quando se consideram ambas as bases. Portanto, este periódico da Saúde pode ser considerado de grande importância na divulgação de pesquisas voltadas aos estudos métricos na área da saúde, no âmbito ibero-americano analisado.

Tabela 1. Produção e impacto geral das revistas do corpus analisado

\begin{tabular}{|c|c|c|c|c|c|c|c|c|c|c|}
\hline & \multirow[b]{2}{*}{ Título da Revista } & \multirow[b]{2}{*}{ ISSN } & \multirow[b]{2}{*}{ País } & \multirow[b]{2}{*}{ Área } & \multicolumn{3}{|c|}{ Scopus } & \multicolumn{3}{|c|}{ Web Of Science } \\
\hline & & & & & $\begin{array}{l}\text { Total de } \\
\text { Artigos } \\
\end{array}$ & $\begin{array}{l}\text { Total de } \\
\text { Citações }\end{array}$ & $\begin{array}{l}\text { Média de } \\
\text { Citações }\end{array}$ & $\begin{array}{l}\text { Total de } \\
\text { Artigos }\end{array}$ & $\begin{array}{l}\text { Total de } \\
\text { Citações }\end{array}$ & $\begin{array}{l}\text { Média de } \\
\text { Citações }\end{array}$ \\
\hline 1 & Gaceta Sanitaria & 0213.9111 & Espanhs & Saúde & 693 & 3224 & 4,65 & 1485 & 2211 & 1,49 \\
\hline 2 & Interciendia & $0378-1844$ & Venezuela & Interdisciplinar & 705 & 1147 & 1,63 & 715 & 890 & 1,24 \\
\hline 3 & Investigación Bibliotecológica & $0187-358 X$ & Méxiळ & Socisis Aplicadss & 137 & 9 & 0,72 & 169 & 64 & 0,38 \\
\hline 4 & Nutricion Hospitalaria & $0212-1811$ & Espanha & Saúde & 1133 & 8147 & 5,42 & 1184 & 4887 & 4,11 \\
\hline 5 & $\begin{array}{l}\text { Perspectivas em Ciência da } \\
\text { Informaçăo }\end{array}$ & 1413.9938 & Brasil & Socisis Aplicadss & 250 & 157 & 0,83 & 281 & 110 & 0,42 \\
\hline 8 & $\begin{array}{l}\text { RAE Revista de Administraçăo de } \\
\text { Empresss }\end{array}$ & 00347590 & Brasil & Sociais Aplicadas & 200 & 338 & 1,68 & 234 & 173 & 0,74 \\
\hline 7 & Revista de Saúde Publica & 00348910 & Brasil & Saúde & 760 & 5941 & 7,72 & 812 & 3378 & 4,18 \\
\hline 8 & Revista Medica de Chile & 07178183 & Chile & Saúde & 1243 & 2888 & 2,18 & 1255 & 1895 & 1,35 \\
\hline 9 & South African Journal of Sdience & 0038.2353 & $\begin{array}{l}\text { Áfica do } \\
\text { Sul }\end{array}$ & Biológicas & 414 & 1479 & 3,57 & 496 & 983 & 1,98 \\
\hline 10 & Transinformaçăo & 0103.3786 & Brasil & Socisis Aplicadss & 79 & 38 & 0,48 & 100 & 38 & 0,38 \\
\hline
\end{tabular}

Fonte: Elaborado pelos autores com base nos dados das bases indexadoras Scopus e WOS, 2016. 
Tabela 2. Produção e impacto das revistas do corpus analisado na temática Estudos Métricos

\begin{tabular}{|c|c|c|c|c|c|c|c|c|c|c|}
\hline & & & & & \multicolumn{3}{|c|}{ Scopus } & \multicolumn{3}{|c|}{ Web Of Science } \\
\hline & Título da Revista & ISSN & País & Área & $\begin{array}{l}\text { Total de } \\
\text { Artigos } \\
\end{array}$ & $\begin{array}{l}\text { Total de } \\
\text { Citações }\end{array}$ & $\begin{array}{l}\text { Média de } \\
\text { Citações }\end{array}$ & $\begin{array}{l}\text { Total de } \\
\text { Artigos }\end{array}$ & $\begin{array}{l}\text { Total de } \\
\text { Citações }\end{array}$ & $\begin{array}{l}\text { Média de } \\
\text { Citações }\end{array}$ \\
\hline$\overline{1}$ & Gaceta Sanitaria & 0213.9111 & Espanha & Saúde & 4 & 12 & 3 & 4 & 9 & 2,25 \\
\hline 2 & Interciencis & $0378-1844$ & Venezuela & Interdisciplinar & 7 & 19 & 2,71 & 7 & 21 & 3 \\
\hline 3 & Investigadón Bibliotecologics & $0187.358 X$ & Méxiø & Socisis Aplicadss & 20 & 7 & 0,35 & 20 & 5 & 0,25 \\
\hline 4 & Nutricón Hospitalaria & 0212-1611 & Espanha & Saúde & 7 & 16 & 2,28 & 7 & 10 & 1,42 \\
\hline 5 & $\begin{array}{l}\text { Perspectivas em Ciênciada } \\
\text { Informaço }\end{array}$ & 1413.9938 & Brasil & Sociais Aplicadas & 21 & 33 & 1,57 & 21 & 31 & 1,47 \\
\hline 6 & $\begin{array}{l}\text { RAE Revista de Administraçăo de } \\
\text { Empresas }\end{array}$ & 00347590 & Brasil & Sociais Aplicadss & 11 & 29 & 2,84 & 11 & 4 & 0,38 \\
\hline 7 & Revista de Saúde Publica & 00348910 & Brasil & Saúde & 10 & 25 & 2,5 & 10 & 18 & 1,6 \\
\hline 8 & Revista Medica de Chile & 07178163 & Chile & Saúde & 2 & 14 & 7 & 2 & 14 & 7 \\
\hline 9 & South African Journsl of Science & $0038-2353$ & $\begin{array}{l}\text { Africa do } \\
\text { Sul }\end{array}$ & Biológicas & 7 & 44 & 6,28 & 7 & 25 & 3,57 \\
\hline 10 & Transinformaçăo & 0103.3788 & Brasil & Socisis Aplicadss & 6 & 8 & 1 & 6 & 5 & 0,83 \\
\hline
\end{tabular}

Fonte: Elaborado pelos autores com base nos dados das bases indexadoras Scopus e Web Of Science, 2016.

Cabe advertir que a diferença no total de artigos por revistas, nas distintas bases de dados, (Tabela 1), foi encontrada também em estudos prévios para periódicos de outras disciplinas. Segundo estes trabalhos, isso poderia ocorrer devido às distintas políticas de indexação das bases de dados, assim como às modificações na cobertura de revistas em distintos períodos analisados, e às limitações resultantes da qualidade dos dados, que podem afetar a representação das produções, especialmente procedentes de países periféricos (Jacsó, 2010; Osca Lluch et al., 2013).

Nota-se que as revistas que apresentam maior impacto de sua produção total em detrimento à produção sobre estudos métricos, tanto na Scopus como na WOS, são: Nutr. Hosp. (Espanha/Saúde), Rev. Saúde Pública (Brasil/Saúde), Interciencia (Venezuela/Interdisciplinar) e Perspect. Ciênc. Inf (Brasil/Sociais Aplicadas). É curioso perceber que a última apresenta sua produção total mais impactante do que a sua produção relativa aos estudos métricos no período (2009-2013), já que, também se caracteriza por publicar pesquisas relacionadas ao domínio de EMI.

Na Tabela 2, sobre o impacto na temática, fica evidente que, com exceção das revistas Interciencia e Revista de Saúde Pública, todas as demais obtiveram maior quantidade de citações na Scopus, sugerindo maior visibilidade nesta última para os artigos que tratam de estudos métricos. Situação semelhante ocorre quando se considera toda a produção científica das revistas e suas citações recebidas, conforme observa-se na Tabela 1 , em relação ao impacto da produção total dos periódicos. Nesse último caso, apenas a revista Transinformação obteve o mesmo impacto mensurado pelas citações nas duas bases de dados.

Quando se mensura o impacto pela média de citações recebidas, no período, pelos periódicos (Figura 1), percebe-se que os resultados para a Gac. Sanit. (Espanha/ Saúde) e a S. Afr. J. Sci. (África/Biológicas) divergem em cada base, sendo que ambas tem maior impacto na Scopus em relação à sua produção total, enquanto que a produção em estudos métricos 
dessas revistas tem maior impacto na WOS.

O periódico RAE (Brasil/Sociais Aplicadas), da Administração, destaca-se pelas citações recebidas em seus artigos em estudos métricos na Scopus (quase seis vezes maior), e pela média de citação geral maior do que em relação à obtida na base WOS, o que denota que seus artigos em estudos métricos tem tido maior visibilidade na primeira base, pois, a Scopus caracteriza-se pela cobertura mais ampla na maioria das áreas do conhecimento.

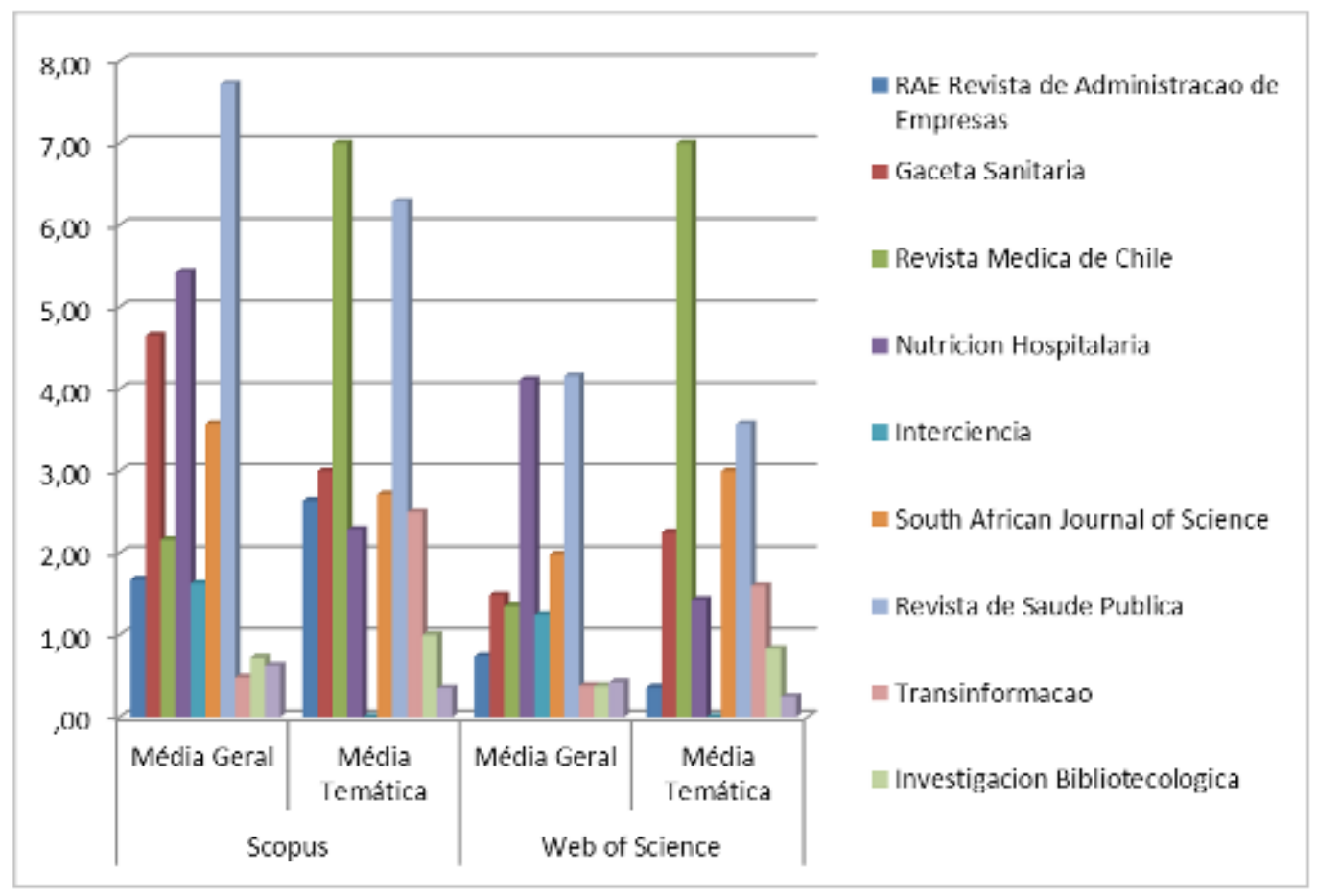

Figura 1. Médias de citação de artigos das revistas do corpus

Fonte: Os autores, 2016.

Ainda, segundo a Figura 1, quando se considera o indicador média de citações, as revistas que se destacam por apresentarem maior impacto em relação aos artigos do domínio de EMI, em ambas as bases, além da Med. Chile (Chile/Saúde), têm-se a Transinformação (Brasil/Sociais Aplicadas) e a Investig. Bibl. (México/Sociais Aplicadas). Dentre as brasileiras de Ciência da Informação, a que se evidencia pela maior média de citação de sua produção total é a Perspectivas em Ciência da Informação, com maior impacto geral do que sua produção no domínio, como já mencionado anteriormente.

O periódico venezuelano, Interciencia, tem a média de citação inexpressiva na temática, apresentando baixo impacto na temática e no geral. O fato de que esta revista é categorizada como interdisciplinar, por um lado pode dispersar a possibilidade de citação em diferentes áreas, devido à ausência de um núcleo científico específico que a consolida, por outro lado pode permitir que a revista tenha visibilidade em diferentes áreas, sendo direcionada a uma gama ampla de pesquisadores de distintas especialidades. Contudo, a partir de 2005, a área de Ciências Sociais na Venezuela apresentou mais artigos indexados na Scopus, conforme dados do Scimago JR (2016). 
Sobre a indexação regional no SciVerse Scopus, 54\% dos periódicos indexados da Scopus são europeus, $32 \%$ da América do Norte e $16 \%$ pertencem a outras regiões como a América Central e do Sul, África, Austrália, entre outras (Vieira, 2013). Essa informação pode explicar o porquê das revistas selecionadas a partir da SciELO apresentarem, em sua maioria, maior impacto na Scopus que na WOS, tanto em relação à produção total das revistas, como em relação à produção sobre estudos métricos da Informação. Ademais, a Scopus apresenta $46 \%$ de títulos de países de outros idiomas que não o inglês (Alves, 2016), o que favorece sua visibilidade nos países iberoamericanos.

Estudos que analisam comparativamente WOS y Scopus concluem que as vantagens de uma ou outra base de dados dependem da área temática específica e também do período temporal da publicação. A esses aspectos, pode-se acrescentar, a partir dos resultados deste estudo, a influência da procedência geográfica das revistas.

Vale dizer também, que a Elsevier, como modo de reconhecer a crescente participação de países latino-americanos nas "publicações mundiais de Ciência e Tecnologia", promoveu, em 2007, o Prêmio Scopus, pelo qual pesquisadores argentinos, colombianos e mexicanos se destacaram. Esse reconhecimento por parte da Editora com a criação dessa premiação anual, também vem contribuindo para que a base ganhe mais visibilidade nos referidos países da América Latina em distintas áreas. (Elsevier, 2007).

Outra explicação para os resultados apresentados decorre das políticas de cada base, que no caso da WOS tem sua amplitude restringida, já que não indexa revistas que tratam do mesmo tipo de escopo. Sobre essa questão, um dos critérios de inclusão de uma revista na WOS é que o conteúdo publicado represente algo novo em uma determinada área, e que não tenha sido explorado por seus outros periódicos ali indexados (Vieira, 2013).

A WOS, além de ter menor cobertura na Saúde quando comparada à Scopus, apresenta sua cobertura distribuída em apenas três grandes áreas, de acordo com a seguinte distribuição: 33\% em Ciências Físicas, 32\% em Ciências da Saúde, 30\% em Ciências Sociais e 20\% em Ciências da Vida, sendo que a classificação em cada uma das áreas não é exclusiva (Vieira, 2013).

Retomando-se a distribuição dos valores de média de citação nos periódicos enfocados no corpus, a Rev. Saúde Pública (Saúde/Brasil) tem média de citação geral maior que a média de citação dos artigos na temática, como já mencionado anteriormente, e apresenta uma visibilidade maior que as outras revistas em relação ao indicador de citação, assim como a Revista Médica do Chile, que, embora se destaque nos estudos métricos pelo alto impacto, é uma das que apresenta maior impacto geral quando comparados os impactos de produção total das outras revistas em ambas as bases, Scopus e WOS.

A seguir, pode-se visualizar a média de citação distribuída por áreas, conforme as áreas em que as revistas são categorizadas. 


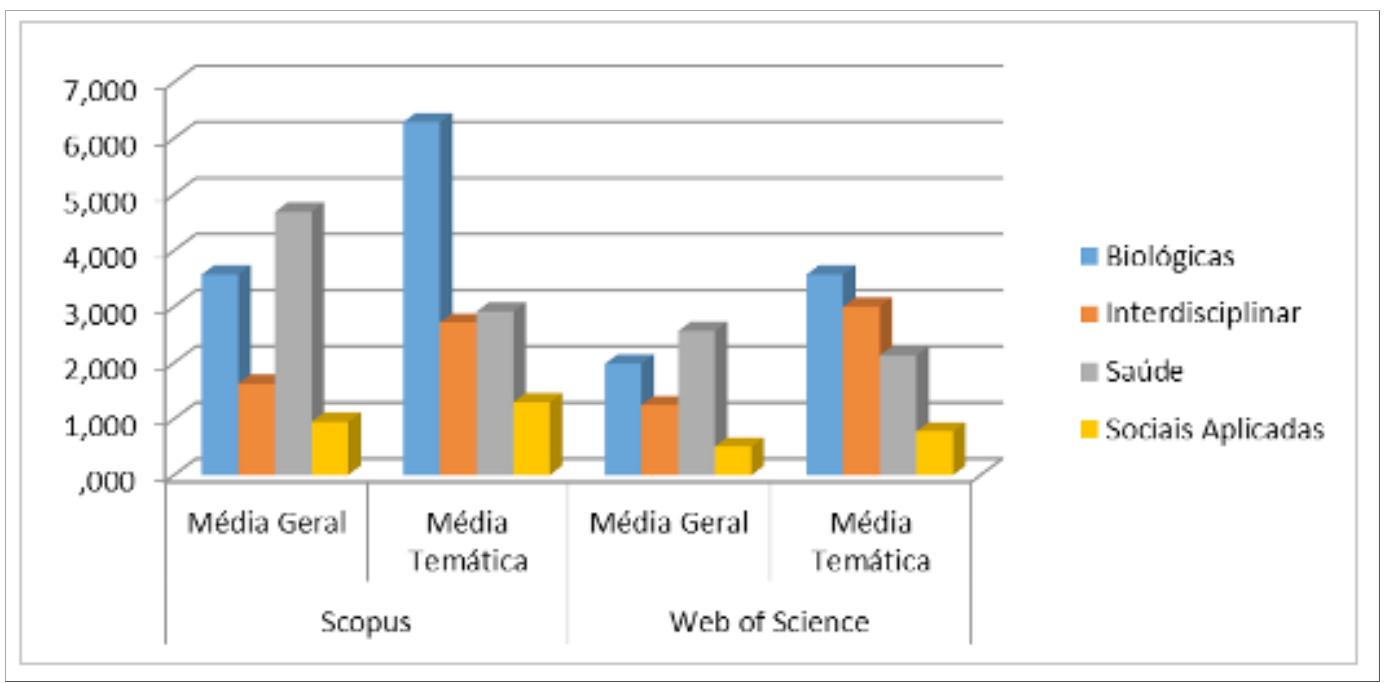

Figura 2. Médias de citação de artigos, por área das revistas, segundo categorias da SciELO Fonte: Os autores, 2016.

Na Figura 2, a única área que apresenta média de citação total maior que a média de citação em estudos métricos é a Saúde, além de ser a área que vem contribuindo expressivamente para a produção e aplicação de estudos métricos da informação, fato ratificado em estudos anteriores relacionados ao domínio (Cabrini Grácio y Tannuri de Oliveira, 2012; Rosas y Freitas, 2016). Isso pode ser explicado pelo fato de que a Saúde historicamente se destaca pelo expressivo volume de sua produção científica.

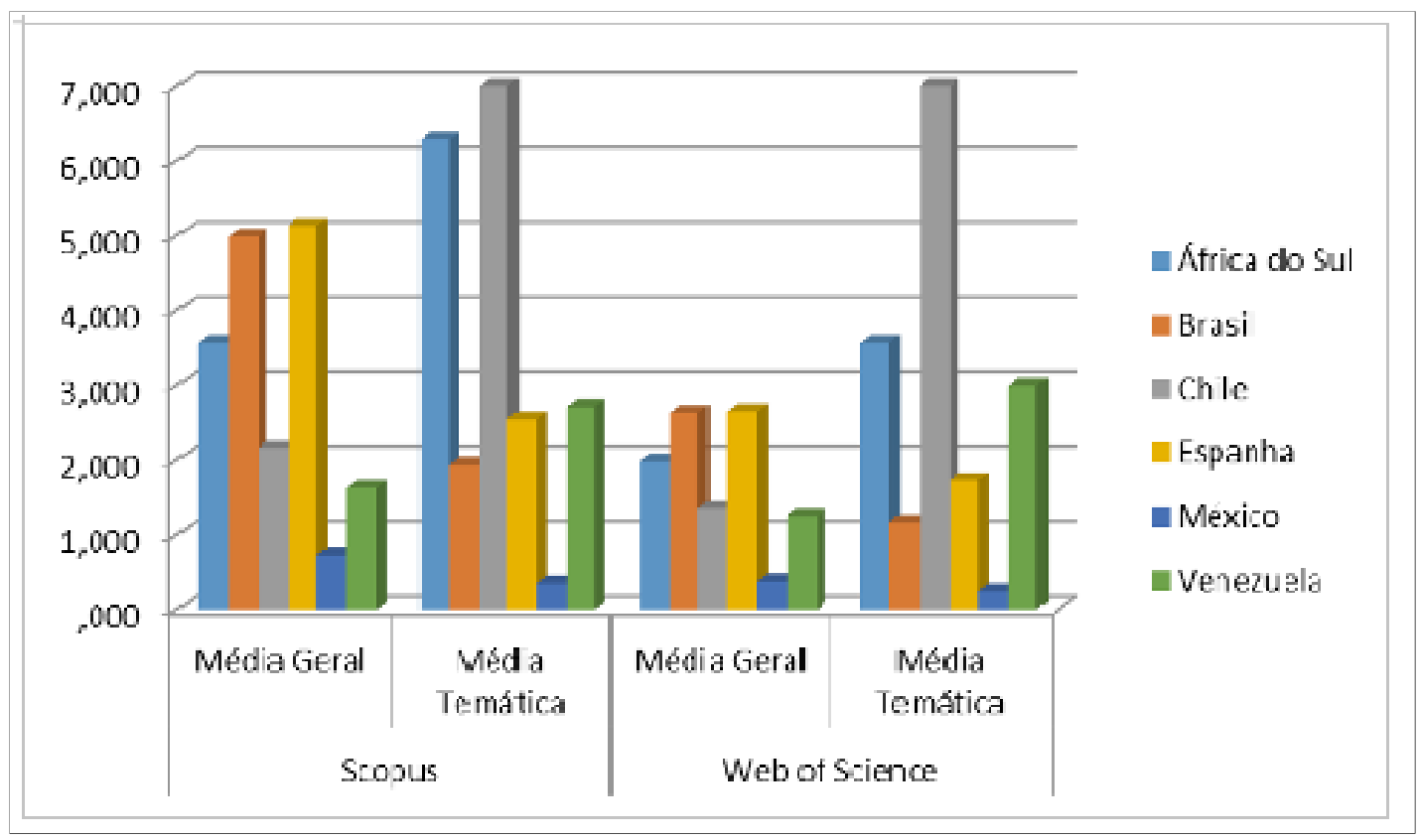

Figura 3. Médias de citação de artigos por países das revistas segundo categorias da SciELO Fonte: Os autores, 2016. 
Em relação aos países (Figura 3), observa-se que Brasil, Espanha e México apresentam impacto de sua produção científica total maior que o impacto dado pela média de citação das suas pesquisas relacionadas aos estudos métricos e Chile, Venezuela e África do Sul, representados pelas revistas Rev. Med. Chile, Interciencia e a S. Afr. J. Sci têm impacto maior em estudos métricos. Mas, vale ressaltar que se considerada a área de Sociais Aplicadas, o Brasil apresenta maior variedade de revistas que publicam sobre a temática, representando $30 \%$ das revistas analisadas no corpus desse estudo. Somando-se à Revista de Saúde Pública (Saúde), esse valor sobe para $40 \%$ do total de revistas analisadas nessa pesquisa, cuja procedência é brasileira.

Com os dados obtidos e analisados, foi possível evidenciar como se configura o espaço destinado aos estudos métricos da informação em diferentes campos do conhecimento, cuja presença no corpus se dá a partir de periódicos indexados na SciELO, Scopus e WOS simultaneamente. Essa configuração não evidencia a rica e vasta produção relacionada aos EMI que se produz no Brasil e em outros países da América Latina e Iberoamérica. Por um lado, os países de origem dos periódicos selecionados são privilegiados por serem representados nessas bases de dados, por outro lado, essa minoria representativa de periódicos e países reflete uma ciência excludente e desigual, que com seus mecanismos de distinção não permite dar visibilidade e acesso à ciência de boa qualidade sobre estudos métricos que se produz em periódicos da $\mathrm{CI}$ e de outras áreas, que não estão indexados nas referidas bases.

\section{Considerações finais}

A pesquisa permitiu analisar a produção periódica científica da Iberoamérica e África do Sul sobre estudos métricos, de modo a observar o seu impacto em nível global, a partir do uso dos indicadores de produção e de citação das bases Scopus e WOS, já que não foi possível obter o indicador de impacto dos artigos do corpus na própria base SciELO, que somente disponibiliza os indicadores de acesso a eles.

Além desse panorama em relação ao impacto total das revistas que publicam sobre estudos métricos, foi possível obter o impacto dos artigos sobre o tema nestas revistas, em ambas as bases, e compará-los. Em síntese observou-se que o impacto dos periódicos, tanto de sua produção total, como a relacionada ao domínio de estudos métricos, é predominantemente mais alto na Scopus em detrimento da WOS, de modo especial devido às políticas e prioridades distintas de cada base. Portanto, foi possível identificar quais as áreas e periódicos que se destacam no contexto dos estudos métricos, não somente de produtividade, mas também de visibilidade científica, e que a Saúde se destaca em ambos os aspectos.

Em síntese, aponta-se que a Saúde é a única área que apresenta média de citação maior de sua produção geral que a média de citação em EMI, mas também é uma área que vem contribuindo expressivamente para a produção e aplicação de estudos métricos da informação. Outra consideração importante é que as Sociais Aplicadas destacam-se, no Brasil, em relação aos estudos métricos, porque congregam periódicos da Ciência da Informação e da Administração no corpus analisado.

O estudo também visou a reflexão sobre a amplitude da cobertura das bases indexadoras mais reconhecidas em âmbito internacional e sobre a importância do uso de seus indicadores. Sugerem-se estudos futuros utilizando os indicadores de citação de bases de dados de acesso aberto como a SciELO, já que, no momento, ainda não estavam disponíveis, apesar de que os indicadores relacionados à altmetria dessa base, já poderiam ser utilizados para apresentar o 
número de acesso aos artigos, a fim de embasar estudos métricos com outras finalidades.

\section{Referências bibliográficas}

Alves, V. (2016). SciVerse Scopus \& avaliações bibliométricas. Apresentação de treinamento Elsevier. Recuperado de http://www.ci.uff.br/ppgci/arquivos/bibliometria\%20SciVerse $\% 20$ Scopus.pdf.

Bornmann, L., y Hans-Dieter, D. (2008). What do citation counts measure?: a review of studies on citing behavior. Journal of Documentation, 64(1), 45-80. doi: $10.1108 / 00220410810844150$

Cabrini Grácio, M.C., y Tannuri de Oliveira, E.F. (2012). A inserção e o impacto internacional da pesquisa brasileira em 'estudos métricos': uma análise na base Scopus. Tendências da Pesquisa Brasileira em Ciência da Informação, 5(1), 1-19. Recuperado de http://hdl.handle.net/11449/114791

Elsevier. (2007). News - América Latina, $03 . \quad$ Recuperado de http://www.loja.elsevier.com.br/bibliotecadigital/news dez07/pdf/edicao03 1210.pdf

Glänzel, W., y Schoepflin, U. (1994). Little scientometrics - Big scientometrics and beyond. Scientometrics, 30(2-3), 375-384. doi: 10.1007/BF02018107

Hicks, D., Wouters, P., Waltman, L., de Rijcke, S. y Rafols, I. (2015). The Leiden Manifesto for research metrics. Nature, 520, 429-431. Recuperado de http://www.ingenio.upv.es/manifiesto

Jacsó, P. (2010). Comparison of journal impact rankings in the SCImago Journal \& Country Rank and the Journal Citation Reports databases. Online Information Review, 34(4), 642-657. doi: $10.1108 / 14684521011073034$

Lu, K. y Wolfram D. (2010) Geographic characteristics of the growth of informetrics literature 1987-2008. Journal of Informetrics, 4(4), 591-601. doi: 10.1016/j.joi.2010.06.008

Machado, R. N. (2007). Análise cientométrica dos estudos bibliométricos publicados em periódicos da área de biblioteconomia e ciência da informação (1990-2005). Perspectivas em Ciência da Informação, 12(3), 2-20. doi: 10.1590/S1413-99362007000300002.

Meneghini, R., y Paker, A. (2010). The extent of multidisciplinary authorship of articles on scientometrics and bibliometrics in Brazil. Interciencia, 35(7), 510-514.

Miguel, S., y Dimitri, P. (2013). La investigación en Bibliometría en la Argentina: quiénes son y qué producen los autores argentinos que realizan estudios bibliométricos en Bibliometría. Información, Cultura y Sociedad, 29, 117-138. Recuperado de http://revistascientificas.filo.uba.ar/index.php/ICS/article/view/677

Mugnaini, R. (2006). Caminhos para adequação da avaliação da produção científica brasileira: impacto nacional versus internacional. (Tese de Doutorado). Universidade de São Paulo, São Paulo, Brasil. doi: 10.11606/T.27.2006.tde-11052007-091052

Nicolaisen, J. (2007). Citation Analysis. Annual Review of Information Science and Technology, 41(1), 609-641. doi: 10.1002/aris.2007.14404101

Osca Lluch, J., Miguel, S., González, C., Peñaranda-Ortega, M., y Quiñones-Vidal, E. (2013). Cobertura y solapamiento de Web of Science y Scopus en el análisis de la actividad científica 
española en psicologia. Anales de Psicología, 29(3), 1025-1031. doi: 10.6018/analesps.29.3.154911

Patra, S.K., Bhattacharya, P., y Verma N. (2006). Bibliometric Study of Literature on Bibliometrics. Bulletin of Information Technology, 26(1), 27-32. doi: 10.14429/dbit.26.1.3672

Porcaro, R M. (2005). Indicadores da sociedade atual - informação, conhecimento, inovação e aprendizado intensivos. A perspectiva da OECD. Data Grama Zero: Revista de Ciência da Informação, 6(4). Recuperado de http://www.datagramazero.org.br/

San Francisco Declaration on Research Assessment (DORA). (2012). Recuperado de http://www.ascb.org/dora/

Rosas, F.S., y Freitas, J.L. (2016). Produção científica em Estudos Métricos da Informação em periódicos presentes nas bases SciELO e Scopus. En $5^{\circ}$ Encontro Brasileiro de Bibliometria e Cientometria (EBBC). São Paulo: USP.

Schoepflin, U., y Glänzel, W. (2001). Two decades of Scientometrics: An interdisciplinary field represented by its leading journal. Scientometrics, 50(2), 301-312. doi: 10.1023/A: 1010577824449

Tannuri de Oliveira, E.F. (2011). Análise de domínio em "Estudos Métricos" no Brasil: produção, impacto e visibilidade em âmbito nacional e internacional. 2013. (Tese de Livre Docência). Universidade Estadual Paulista Júlio de Mesquita Filho, Marília-SP, Brasil.

Vieira, E.S. (2013). Indicadores bibliométricos de desempenho científico: estudo da aplicação de indicadores na avaliação individual do desempenho científico. (Tese de Doutorado). Universidade do Porto, Porto, Portugal. 